\title{
Multiple Perceptible Watermarking for Copyright Protection
}

\author{
V. Mehan \\ NIT Jalandhar \\ Punjab \\ India
}

\author{
R. Dhir \\ NIT Jalandhar \\ Punjab \\ India
}

\author{
Y. S. Brar \\ GNDEC Ludhiana \\ Punjab \\ India
}

\begin{abstract}
Perceptible watermarking system is an imperative copyright protection tool for digital images. Perceptible watermarks safeguard the digital images in a better vigorous manner, then imperceptible watermarks. The paper presents a multiple perceptible watermarking approach for copyright protection. Two perceptible watermarks are embedded to the color digital images. The watermarked image comprehends noticeable but un-obstructive watermark evidence. The proposed system can be castoff for advertisement and copyright uses.
\end{abstract}

\section{General Terms}

Watermarking

\section{Keywords}

Watermarking; Perceptible Watermarking; Imperceptible Watermarking; Copyright Protection.

\section{INTRODUCTION}

Intellectual property rights (IPR) included watermarking practice under the data hiding process and classified them [1] as shown in the Figure 1 below:

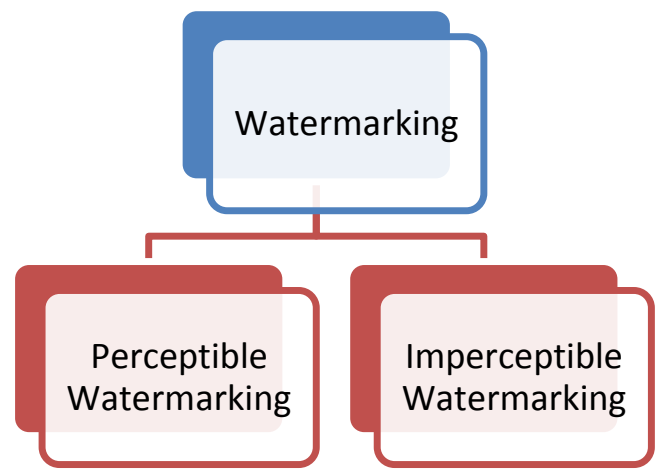

Fig 1: Types of Watermarking

Perceptible watermark is a secondary translucent image overlaid on the primary image [2]. Perceptible watermarks are used in much the same way as their bond paper ancestors, where the opacity of paper is altered by physically stamping it with an identifying pattern.

Perceptible watermarks are an extension of the concept of logos. Such watermarks are applicable to images only. These logos are inlaid into the image but they are transparent. Such watermarks cannot be removed by cropping the center part of the image. Further, such watermarks are protected against attacks such as statistical analysis. Stock Photography agencies often add a watermark in the shape of a copyright symbol ("๑") to previews of their images, so that the previews do not substitute for high-quality copies of the product included with a license.

Perceptible watermarks change the signal altogether such that the watermarked signal is totally different from the actual signal, e.g. adding an image as a watermark to another image. For example, perceptible watermarks, as visible watermarks, are used in much the same way as their paper ancestors - to identify the source or ownership of a document.

Perceptible watermarks are especially useful for conveying an immediate claim of ownership [3]. The main advantage is that they virtually eliminate the commercial value of the document to a would-be thief without lessening the document's utility for legitimate purposes. You can add a color transparent perceptible watermark to your digital images and photos. The watermark can be your copyright or the URL of your site or your logo. The watermarking tool is useful for computer painters, designers, and banner makers, who send their works to a customer through the Internet for evaluation.

The drawbacks of perceptible watermarks are degrading the quality of image and detection by visual means only [4]. Thus, it is not possible to detect them by dedicated programs or devices. Such watermarks have applications in maps, graphics and software user interface.

Imperceptible watermarks hide information invisibly in documents [5]. The term data hiding will be used when the imperceptible watermarks themselves contain data. Imperceptible watermarks that are unknown to the end user are steganographic. Imperceptible watermarks are used to track the images, documents or videos to its original owner.

\section{LITERATURE REVIEW}

Attacking visible watermarking scheme is proposed by Huang and $\mathrm{Wu}$ [6]. Perceptible watermarking structures can be very beneficial in allocating images with copyrights on Internet. Probable security glitches and attacking structures are also encompassed by the authors.

Luo et al. proposed a technique of joint halftoning and visible watermarking [7]. Digital half-toning is a method to transform continuous tone images into two tone images. Anticipated features of perceptible watermarking are discussed in this paper.

Chuang et al. proposed a scheme of unseen visible watermarking [8]. The scheme retains benefits of both perceptible and imperceptible watermarking systems.

\section{PROPOSED APPROACH}

Algorithm for embedding copyright message and watermark image is shown in Figure 2. 


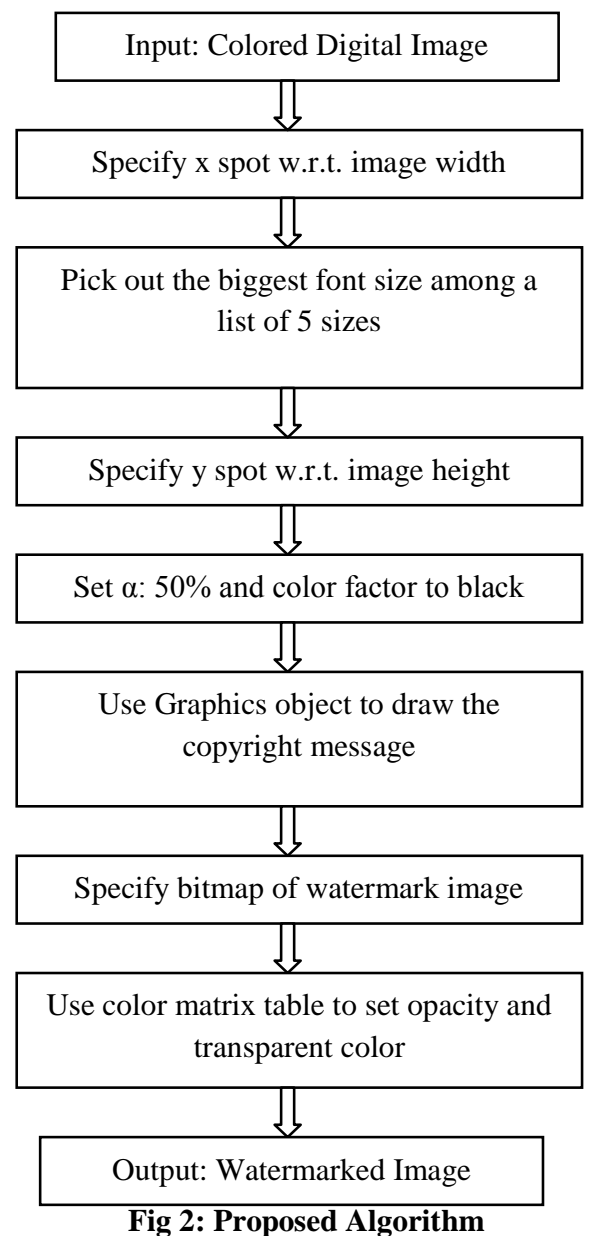

\section{EXPERIMENTAL RESULTS}

A set of 25 images are used for watermarks inclusion as shown in Table 1. Dimension of every image is taken to be 512x512. Implanted watermark consist of copyright image and copyright message. Combination of both copyright image and message produces 25 diverse watermarks. PSNR quality parameter is evaluated for each image with varying watermark size in range of 400-576 for image and font range of 4-8 for message. Insertion Computing Time (ICT) and Extraction Computing Time (ECT) parameters are measured to determine the computing time while implanting and recovering of watermarks.

Table 1: PSNR results for $512 \times 512$ images

\begin{tabular}{|cccc|}
\hline Image & PSNR(R) & PSNR(G) & PSNR(B) \\
\hline Image1 & 49.65 & 44.93 & 45.27 \\
\hline Image2 & 45.35 & 46.22 & 45.86 \\
\hline Image3 & 41.56 & 40.71 & 39.63 \\
\hline Image4 & 51.31 & 45.95 & 42.52 \\
\hline Image5 & 57.12 & 45.17 & 45.69 \\
\hline Image6 & 45.87 & 46.59 & 40.3 \\
\hline
\end{tabular}

\begin{tabular}{|cccc|}
\hline Image7 & 41.1 & 41.25 & 46.58 \\
\hline Image8 & 39.76 & 40.8 & 41.01 \\
\hline Image9 & 38.74 & 40.63 & 41.73 \\
\hline Image10 & 41.62 & 40.3 & 39.08 \\
\hline Image11 & 43.5 & 44.65 & 45.14 \\
\hline Image12 & 53.13 & 44.06 & 40.55 \\
\hline Image13 & 46.67 & 45.41 & 45.79 \\
\hline Image14 & 50.03 & 44.61 & 42.35 \\
\hline Image15 & 42.31 & 43.13 & 40.37 \\
\hline Image16 & 51.88 & 44.37 & 40.3 \\
\hline Image17 & 41.85 & 43.47 & 39.35 \\
\hline Image18 & 41.37 & 41.76 & 41.38 \\
\hline Image19 & 38.76 & 39.57 & 38.99 \\
\hline Image20 & 36.02 & 41.64 & 44.13 \\
\hline Image21 & 44.75 & 45.13 & 45.38 \\
\hline Image22 & 40.12 & 42.35 & 39.65 \\
\hline Image23 & 43.5 & 44.36 & 42.86 \\
\hline Image24 & 37.64 & 38.66 & \\
\hline Image25 & 42.68 & 40.84 & \\
\hline
\end{tabular}

Minimum $\left(\mathrm{M}_{\mathrm{N}}\right)$ and maximum $\left(\mathrm{M}_{\mathrm{X}}\right)$ PSNR parameter values obtained are shown in Table 2. Average (AG) PSNR parameter values using complete set of test images are displayed in Table 3.

Table 2: $M_{N}$ and $M_{X}$ PSNR values

\begin{tabular}{|c|c|c|c|c|c|}
\hline \multicolumn{3}{|c}{ PSNR(R) } & \multicolumn{2}{c|}{ PSNR(G) } & \multicolumn{2}{c|}{ PSNR(B) } \\
\hline $\mathrm{M}_{\mathrm{N}}$ & $\mathrm{M}_{\mathrm{X}}$ & $\mathrm{M}_{\mathrm{N}}$ & $\mathrm{M}_{\mathrm{X}}$ & $\mathrm{M}_{\mathrm{N}}$ & $\mathrm{M}_{\mathrm{X}}$ \\
\hline 36.02 & 57.12 & 38.66 & 46.59 & 38.07 & 46.58 \\
\hline
\end{tabular}

Table 3: $\mathbf{A}_{\mathrm{G}}$ PSNR values

\begin{tabular}{|c|c|c|}
\hline $\mathrm{A}_{\mathrm{G}}$ PSNR(R) & $\mathrm{A}_{\mathrm{G}} \operatorname{PSNR}(\mathrm{G})$ & $\mathrm{A}_{\mathrm{G}}$ PSNR(B) \\
\hline 44.31 & 43.15 & 42.16 \\
\hline
\end{tabular}


Comparative analysis of PSNR for R, G, B and MPSNR w.r.t. varying watermark size for $512 \times 512$ images is presented in Figure 3. Comparative analysis of ICT and ECT for each test image is displayed in Figure 4.

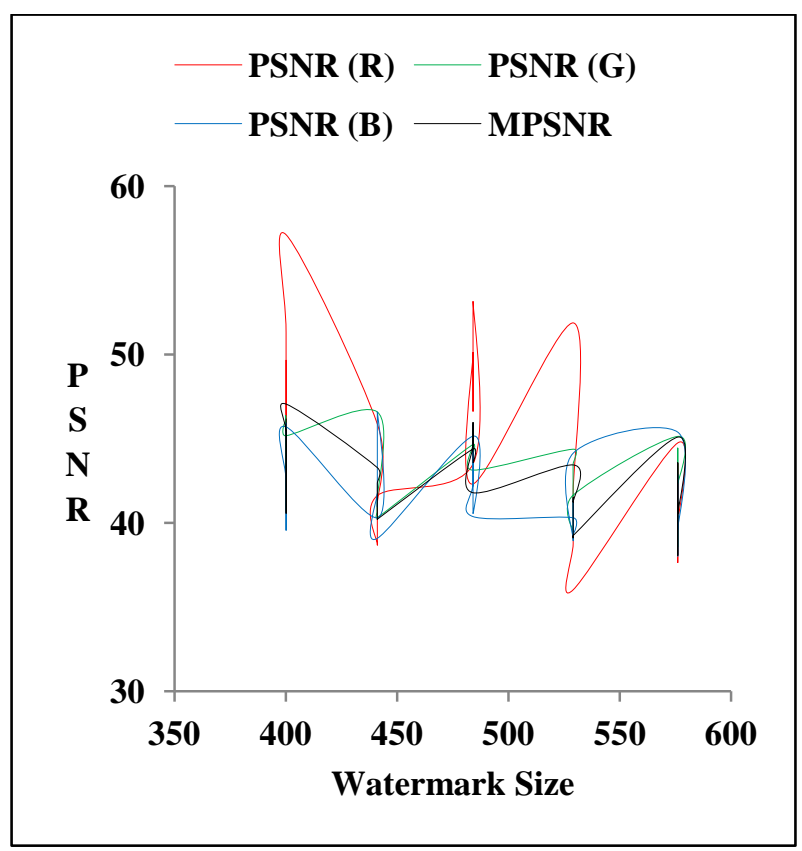

Figure 3: PSNR Comparisons

Correlation Coefficient (CC) parameter identifies the association among two images. The $\mathrm{CC}$ between original image and watermarked image computes image deformation at pixels level. $\mathrm{CC}$ is measured for all $512 \times 512$ images with varying watermarks size as shown in Table 4.

Our approach generates a high positive $\mathrm{CC}$ which reveals a strong association among host image and watermarked image. A positive correlation creates a $\mathrm{CC}$ value close to +1 . Experimental results state an improvement of $\mathrm{CC}$ in comparison to the previous approaches. Comparative Analysis of CC w.r.t. varying watermark size is shown in Figure 5.

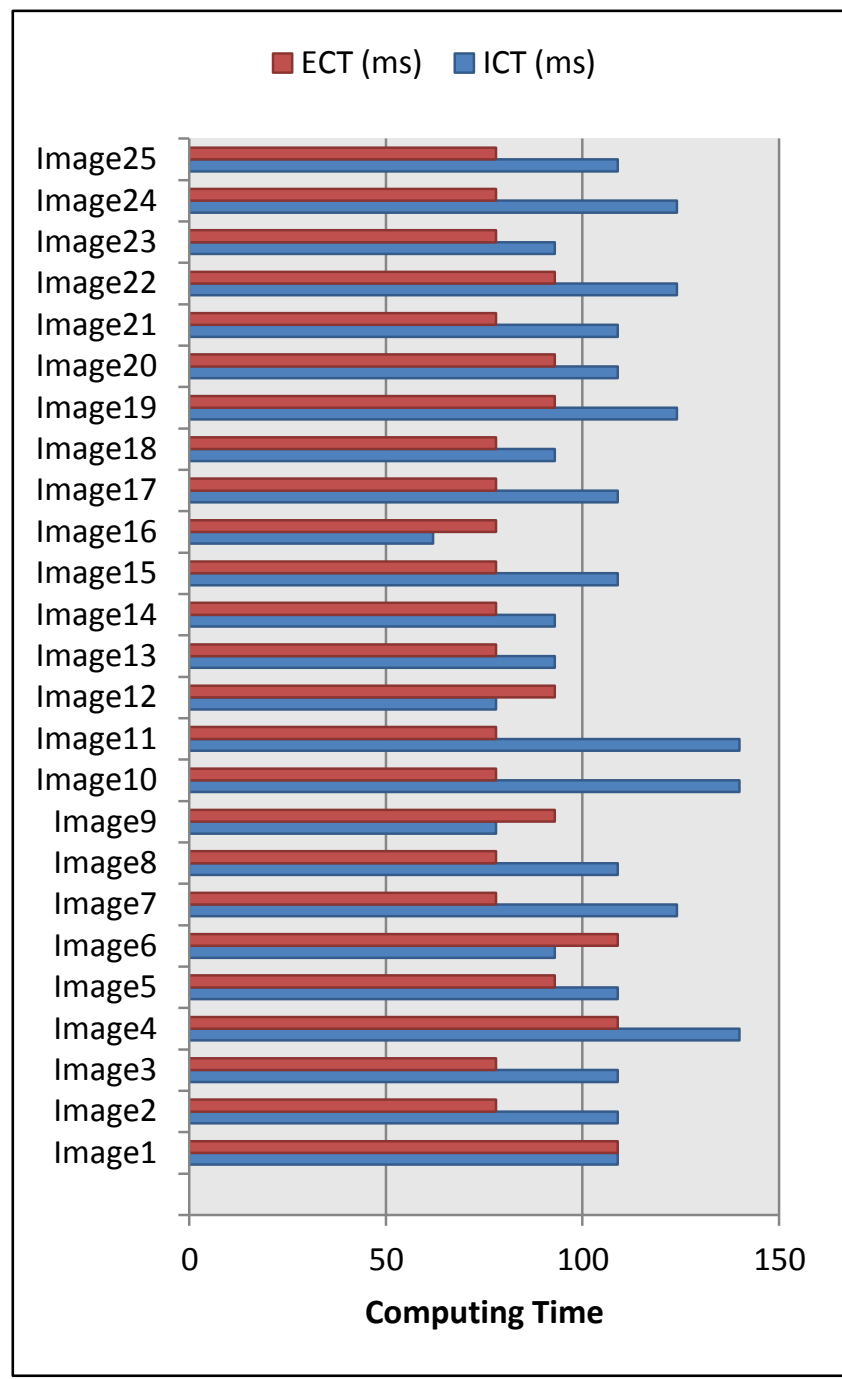

Fig 4: Computing Time Evaluations

Table 4: CC results for $512 \times 512$ images

\begin{tabular}{|ccc|c|}
\hline Image & $\mathrm{CC}(\mathrm{R})$ & $\mathrm{CC}(\mathrm{G})$ & $\mathrm{CC}(\mathrm{B})$ \\
\hline Image1 & 0.99984 & 0.99962 & 0.99912 \\
\hline Image2 & 0.99969 & 0.99964 & 0.99976 \\
\hline Image3 & 0.99913 & 0.99869 & 0.99862 \\
\hline Image4 & 0.99987 & 0.99985 & 0.99904 \\
\hline Image5 & 0.99999 & 0.99976 & 0.99985 \\
\hline Image6 & 0.99964 & 0.99979 & 0.99921 \\
\hline Image7 & 0.99900 & 0.99930 & 0.99988 \\
\hline Image8 & 0.99917 & 0.99930 & 0.99943 \\
\hline Image9 & 0.99894 & 0.99891 & 0.99922 \\
\hline Image10 & 0.99967 & 0.99956 & 0.99921 \\
\hline
\end{tabular}




\begin{tabular}{|cccc|}
\hline Image11 & 0.99972 & 0.99973 & 0.99987 \\
\hline Image12 & 0.99995 & 0.99976 & 0.99937 \\
\hline Image13 & 0.99978 & 0.99967 & 0.99973 \\
\hline Image14 & 0.99981 & 0.99940 & 0.99954 \\
\hline Image15 & 0.99945 & 0.99958 & 0.99923 \\
\hline Image16 & 0.99985 & 0.99931 & 0.99178 \\
\hline Image17 & 0.99949 & 0.99940 & 0.99889 \\
\hline Image18 & 0.99895 & 0.99898 & 0.99930 \\
\hline Image19 & 0.99795 & 0.99817 & 0.99917 \\
\hline Image20 & 0.99734 & 0.99912 & 0.99986 \\
\hline Image21 & 0.99967 & 0.99963 & 0.99963 \\
\hline Image22 & 0.99927 & 0.99963 & 0.99934 \\
\hline Image23 & 0.99965 & 0.99972 & 0.99969 \\
\hline Image24 & 0.99906 & 0.99905 & 0.99914 \\
\hline Image25 & 0.99958 & 0.99939 & 0.99964 \\
\hline
\end{tabular}

safeguarding copyright protection of digital images. Future work should focus on the design of robust insertion and extraction of multiple watermark content against geometric attacks.

\section{REFERENCES}

[1] Joshi, A. M. and A. Darji (2009). "Efficient Dual Domain Watermarking Scheme for Secure Images". International Conference on Advances in Recent Technologies in Communication and Computing, pp: 909-914

[2] Wei-Fan, H. and L. Pei-Yu "Imperceptible Visible Watermarking Scheme Using Color Distribution Modulation". 9th International Conference on Ubiquitous Intelligence \& Computing and 9th International Conference on Autonomic \& Trusted Computing, pp: 1002-1005.

[3] Samuel, S. and W. T. Penzhorn (2004). "Digital watermarking for copyright protection". 7th AFRICON Conference in Africa, pp: 953-957.

[4] Johnson, N. F. (1999). "An Introduction to Watermark Recovery from Images". Proceedings of the SANS Intrusion Detection and Response Conference, San Diego, CA, pp: 9-13.

[5] Takeuchi, S., A. Kunisa, K. Tsujita and Y. Inoue (2005). "Geometric distortion compensation of printed images containing imperceptible watermarks". International Conference on Consumer Electronics, pp: 411-412.

[6] Chun-Hsiang, H. and W. Ja-Ling. "Attacking visible watermarking schemes". IEEE Transactions on Multimedia, Vol. 6, No. 1, 2004, pp: 16-30.

[7] Hao, L., P. Jeng-Shyang, L. Zhe-Ming and L. Bin-Yih (2006). "Joint Halftoning and Visible Watermarking". International Conference on Intelligent Information Hiding and Multimedia Signal Processing, pp: 109-112.

A multiple perceptible watermarking technique for color digital images is proposed in this paper. The paper gives an overview to elementary necessities of perceptible watermarking system. PSNR and CC parameters evaluation determines the effectiveness of the proposed approach. Perceptible watermarking plays a significant factor in

[8] Shang-Chih, C., H. Chun-Hsiang and W. Ja-Ling (2007). "Unseen Visible Watermarking". IEEE International Conference on Image Processing, pp: 261-264. 